[2] Diederichsen, LP, et al., Cardiac Abnormalities in Adult Patients With Polymyositis or Dermatomyositis as Assessed by Noninvasive Modalities, Arthritis Care Res (Hoboken). 2016 Jul;68(7):1012-20

Disclosure of Interests: None declared

DOI: 10.1136/annrheumdis-2019-eular.7757

\section{FRI0686 RELATIONSHIP BETWEEN VITAMIN D SUPPLEMENTATION AND MUSCULAR STRENGTH IN ELDEST POPULATION. A SISTEMATIC REVIEW}

Maria Betina Nishishinya ${ }^{1}$, Claudia Alejandra Pereda Testa ${ }^{2}$, Adela Cristina $\mathrm{Cis}^{3}$, Monica Solana-Tramunt ${ }^{4}$, M Guerra-Balic ${ }^{4} .{ }^{1}$ Instituto Traumatológico Quirón,

Barcelona, Programa de doctorado en Ciencias de la Educación y el Deporte de la Facultat de Psicologia, Ciències de l'Educació i de l'Esport Blanquerna,Barcelona, Rheumatology, Barcelona, Spain; ${ }^{2}$ Hospital HLA Mediterraneo, Almería, Rheumatology, Almería, Spain; ${ }^{3}$ Hospital HLA Mediterraneo, Almería, Sport Medicine, Almería, Spain; ${ }^{4}$ Ramon Llull University, FPCEE Blanquerna, Barcelona, Department of Sports Sciences, Barcelona, Spain

Background: Sarcopenia is the loss of skeletal muscle mass, strength and function that occurs as a consequence of aging. This condition result in physical disability, which limits the capacity to walk, increases the risk of falls and osteoporotic fractures. Several studies suggested an inverse relation between $25 \mathrm{OHD}$ serum levels, muscular strength and physical performance in the eldest.

Objectives: To evaluate if vitamin D supplementation in patients > 50 years improves muscle mass, strength and performance in older patients. Methods: We performed a systematic review through Medline, Cochrane Library, and EMBASE. Inclusion criteria: 1) patients > 50 years old, 2) receiving treatment with vitamin $D$ 3) muscle mass and muscle strength measurements 4) systematic reviews (SR) 5) randomized clinical trials (RCT) 6) Papers written in English or Spanish.

Results: Five studies were included (4 SR and $1 \mathrm{RCT}$ ), $\mathrm{n}$ patients ranged from 121 to 5615. Patients mean age oscillated between 58-88 years, receiving variable vitamin $D$ dose. Follow-up period fluctuated from 6 to 12 months.

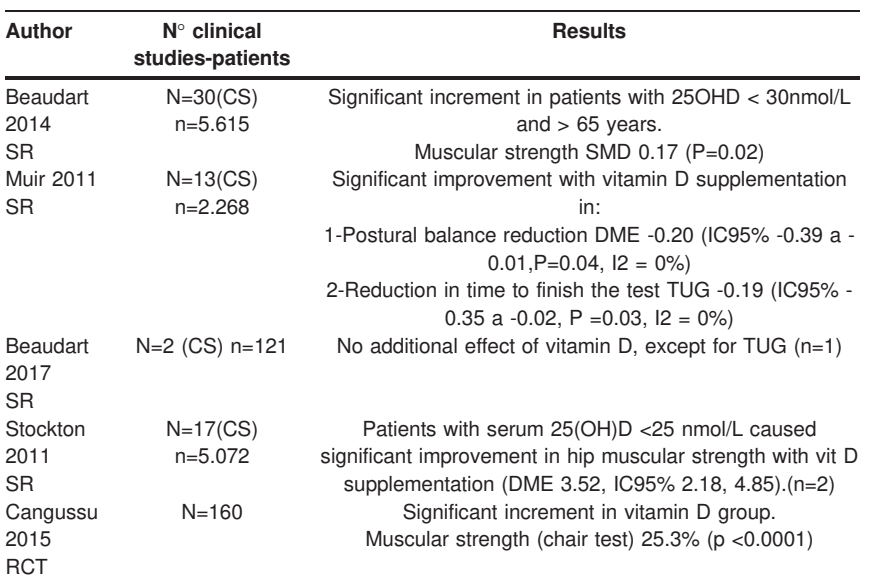

$\mathrm{CS}=$ Clinical studies, $\mathrm{SR}=$ Systematic reviews, $\mathrm{RCT}=$ Randomized clinical trials,

$\mathrm{SMD}=$ Standardized mean difference, $\mathrm{TUG}=$ Time UP and Go.

Conclusion: - Vitamin D supplementation in patients > 65 years with inadequate $25 \mathrm{OHD}$ serum levels, improve muscular strength.

- There were no benefits in muscle mass or muscular performance.

\section{REFERENCES:}

[1] Beaudart C, Dawson A, Shaw S C, Harvey N H, Kanjis J A, Binkley N, et al. Nutrition and Physical activity in the prevention and treatment of sarcopenia.: systematic review. Osteopor Int 2017; 28: 1817-1833.

[2] Cangussu LM, Nahas-Neto J, Orsatti CL, Bueloni-Dias FN, Nahas EAP. Effect of Vitamin D supplementation alone on muscle function in postmenopausal women: a randomized double-blind, placebo-controlled clinical trial. Osteopor Int 2015; 26:2413-2421.

\section{FRI0687 NEUROMYELITIS SPECTRUM DISORDERS ASSOCIATED WITH AUTOIMMUNE DISEASES: DIFFERENCES IN CLINICAL CHARACTERISTICS AND MRI FINDINGS}

Milena Rodriguez Alvarez ${ }^{1}$, Manjeet Bhamra ${ }^{1}$, Vinodkumar Velayudhan ${ }^{1}$, Fernando. Cuascut ${ }^{2}$, Latoya Freeman ${ }^{1}$, Su Yien Zhaz ${ }^{3}$, Joshy Pathiparampil ${ }^{1}$, Naureen Kabani ${ }^{1}$, Kristaq Koci ${ }^{1}$, Alexandra Kreps ${ }^{1}$, Justin Levinson ${ }^{4}$, Sophia Francis ${ }^{1}$, Steve Xie ${ }^{1}$, Helen Valsamis ${ }^{1}$, Abhimanyu Amarnani ${ }^{1}$ David Ozeri', Samir Alkabie ${ }^{1}$, Yaacov. Anziska', Isabel Mcfarlane ${ }^{1} .{ }^{1}$ SUNY Downstate Medical Center, Medicine, BROOKL YN, United States of America; ${ }^{2}$ Baylor University Medical Center, Neurology, Dallas, United States of America; ${ }^{3}$ Samaritan Medical Center, Watertown, United States of America; ${ }^{4}$ Temple University Hospital, Philadelphia, United States of America; ${ }^{5}$ Sheba Medical Center, Tel Aviv, Israel

Background: Neuromyelitis Optica Spectrum disorders (NMOSD) is a rare autoimmune disease characterized by optic neuritis (ON) and/or longitudinal extensive transverse myelitis (LETM). It is commonly associated with other autoimmune diseases (OAD). Recent reports suggested racial differences in clinical phenotype and presentation of NMOSD. However, data on Black population is scarce.

Objectives: We aim to characterize, in our largely Black population, the clinical, laboratory and radiologic features of patients with NMOSD and OAD. We also aim to ascertain differences in clinical presentation between NMOSD patients with and without OAD.

Methods: In a retrospective analysis, patients $\geq 18$ years of age with a confirmed diagnosis of NMOSD as per the International Panel for NMOSD Diagnosis Criteria, seen at 2 NYC urban hospitals from 1/2005 to $4 / 2017$ were identified. Demographic, clinical, and laboratory data were extracted together with expanded disability status scales (EDDS) and imaging studies. Brain magnetic resonance imaging (MRI) was reviewed by a neuro-radiologist who applied the NMOSD Radiological criteria to identify typical findings of the disease.

Results: Forty-one patients fulfilled NMOSD criteria. $85.4 \%$ were women with a mean age of $44.7 \pm 2.03$ years. $82.9 \%$ of the patients were Black and $34.1 \%(14 / 41)$ had an associated OAD. Systemic lupus erythematosus (SLE) was the most common OAD present prior to NMOSD diagnosis, followed by thyroid disease and Sjogren's syndrome. Aquaporin 4 immunoglobulin G (AQP4lgG) was positive in $82.9 \%$ of the entire cohort and in $76.9 \%(10 / 13)$ of patients with NMOSD and OAD. Hypertension $(33.3 \%$ vs. $15.3 \%)$, and cardiovascular disease $(13.3 \%$ vs. $4 \%)$ were more frequent in NMOSD with OAD, compared with the NMOSD only group. On initial presentation of the NMOSD only group, visual changes $(40 \%$ vs. $28.5 \%)$ and ON $(38.4 \%$ vs. $20 \%)$ were predominant. In the intial presentation of NMOSD with OAD group, sensory loss $(78.5 \%$ vs $57.7 \%$ ), acute myelitis ( $40 \%$ vs. $23.1 \%$ ), and elevated C reactive protein (CRP) $(20.85 \pm 11.2$ vs. $3.2 \pm 1.85 \mathrm{mg} / \mathrm{d} / \mathrm{L})$ were more freuent. Disability scores (EDDS) were 5.5 for each group. Brain MRI revealed lesions affecting corpus callosum in a marble pattern, $(21.4 \%$ vs. $13.6 \%)$, the hemispheres in a spindle like pattern(33\%vs $22.7 \%)$, the dorsal medulla $(50 \%$ vs. $39.1 \%)$, the area postrema $(38.5 \%$ vs. $27.3 \%)$ and the pons (21.4\% vs. 13.4) for NMOSD with OAD and without respectively. LETM with predilection for the thoracic region was $(66.7 \%$ vs $54.5 \%)$, cord edema $(69.2 \%$ vs. $40.9 \%)$ and gadolinium enhancement $(69.2 \%$ vs. $59.1 \%)$ for NMOSD with OAD and NMOSD only patients respectively. Conclusion: AQP4lgG-positivity was observed in most of the cases in our predominantly Black NMOSD population. Over a third of the NMOSD patients had OAD. SLE was the most commonly reported. NMOSD with OAD patients tended to present with sensory loss, acute myelitis, and elevated CRP, while in NMOSD without OAD presented more with visual changes and ON. The NMOSD with OAD group had more MRI lesions involving corpus callosum, hemispheres, brainstem and LETM, compared to those with NMOSD only group.

REFERENCES:

[1] Katz S I. NMOSD. Multiple Sclerosis and Other Demyelinating Diseases $p$. 864896. June 2016, Vol.22, No.3. Doi: 10.1212/CON.0000000000000337

Disclosure of Interests: None declared

DOI: 10.1136/annrheumdis-2019-eular.6432 